\title{
Multipoint Real-time Monitoring System of Household Air Quality Based
}

\section{on ZigBee}

\author{
YIN Xin-tong, NI Jian-yun, QI Lei ,QIU Zhuang-zhuang and WANG Yi-ru \\ Tianjin Key Laboratory for Control Theory \& Applications in Complicated Systems, School of \\ Electrical and Electronic Engineering, Tianjin University of Technology, Tianjin 300384 \\ 1427521126@qq.com, nijianyun@tjut.edu.cn, 1576705909@qq.com, 15222563126@qq.com, \\ 2591152263@qq.com
}

Keywords: ZigBee wireless sensor network (WSN) CC2530 household air quality monitor

\begin{abstract}
.
Nowadays, with the improvement of buildings leakprofness and indoor decoration materials' widely use in sealing, household air quality has been the object of attention. So we combine the ZigBee wireless communication technology with sensor detection technology and a set of multipoint real-time monitoring system of household air quality based on ZigBee is designed. CC2530 chip microprocessor as the core, system adopt master-slave structure. Its sensor nodes from the machine collect air environmental data, air temperature and humidity sensor nodes to real-time transmission to the host gateway nodes and summary. Master machine combined with monitoring data to determine and display the whole household air quality status, so as to realize the state monitor alarm, and then take measures to improve air quality.
\end{abstract}

\section{Introduction}

Nowadays, household air quality attracts widespread attention. $80 \%$ of the time people work, study and rest indoors, so household environment quality directly affect people's health. In addition, the worldwide energy requirements and the improvement of buildings leakprofnes reduce indoor and outdoor air circulation accordingly. Most of the decoration materials, interior decoration and indoor facilities are synthesized by organic. The spread of volatile organic compounds gas worsen indoor air quality makes the influence of the indoor air quality of human life, the health been higher than outdoor air quality [1]. At present, the domestic air environment detection method mainly has two kinds: one kind is artificial sampling laboratory analysis. This method of equipment requirements is low and the operation is relatively simple, but the whole detection process takes so longer that can't meet the requirement for real-time monitoring of air environment. Another kind is the online test. Although this method has the advantage of high detection accuracy, high resolution and sensitivity, it mainly relies on imported equipment and can't provide a strong guarantee for the full and accurate detection of atmospheric environment [2]. So through the analysis on indoor air quality monitoring requirements and implementation methods, this topic designs a set of multipoint real-time monitoring system of household air quality based on ZigBee, which operation is simple and has a good real-time performance.

The system includes three parts: air quality test detection slave, ZigBee wireless sensor network and the monitor system of the main control machine. ZigBee network has three types of network topology: the topology of the network topology, tree network topology and network topology. Tree 
network topology is easy to extend and fault isolation is easier, but the nodes are so dependent on the root that the whole network will not work properly if the root fails; The terminal nodes of a star network topology need to be within the scope of the gateway node, thereby limiting the network coverage area; the network topology has the advantages of covering a wide area, but also has the disadvantages of managing complexity and short battery life [3]. ZigBee is characterized by close range, low complexity, self-organization, low power consumption, low data rate and low cost [4]. This system is applied to the general residence because of its small monitoring scope, so we choose the star network topology structure to form the ZigBee wireless communication network.

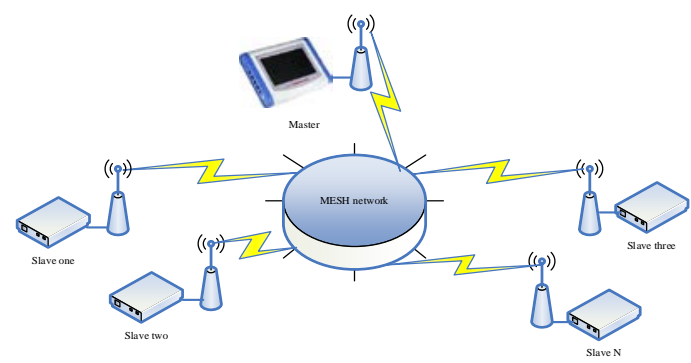

Fig.1 System structure

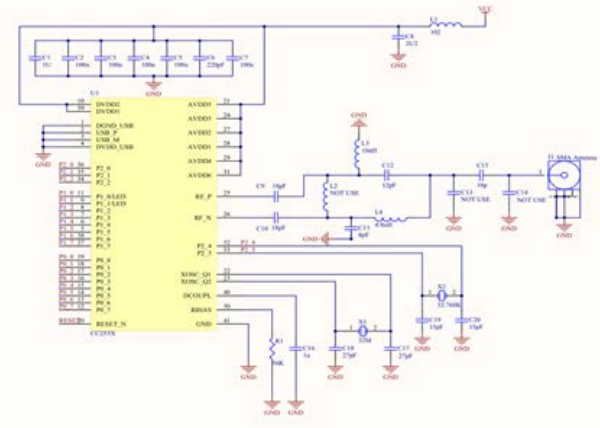

Fig.3 CC2530 circuit

\section{System hardware}

\subsection{Detection slave hardware}

The main function of detecting terminal nodes is to detect the contents of $\mathrm{CO}, \mathrm{CO}_{2}$, air smoke content, temperature and humidity in indoor air, and detected data will be send to host detection software through ZigBee wireless communication network. Detection slave mainly includes CPU module, temperature and humidity, gas sensor module, power module, alarm limits module, wireless communication module, LED display circuit and switch circuit.

When detection slave works, the sensors will detect the data through signal transformation and A/D conversion, which will be read by the microprocessor and sent to the host detection software via ZigBee wireless mode; LCD display module online displays the current air quality level; LED display circuit is used to show whether the device is working properly, the communication is normal, the function of the gas overvalue alarm. The hardware structure is shown in figure 2.

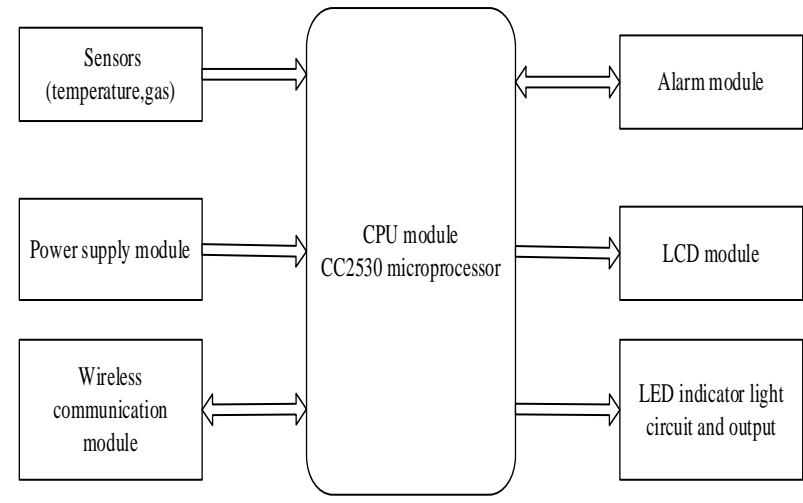

Fig.2 Hardware structure of slave

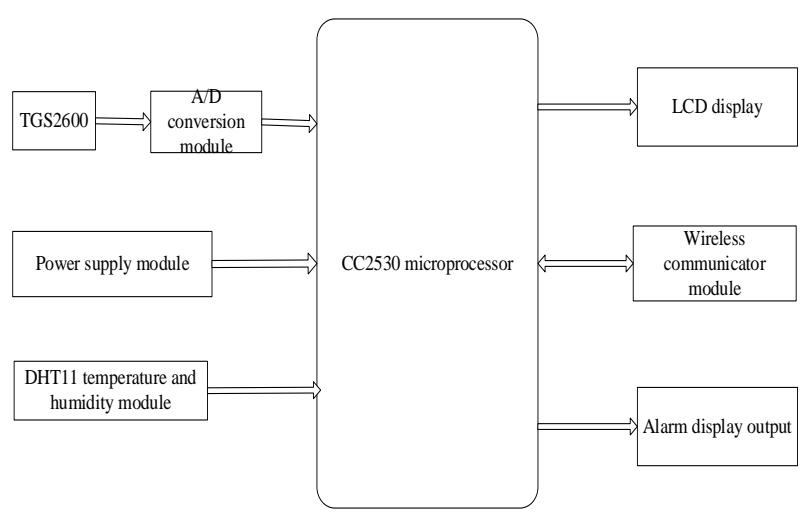

Fig.4 Hardware structure of master

Put the CC2530 as microprocessors, CPU module uses the TI company's CC2530 chip as the controller. CC2530 is a real tablet solution used for the 2.4-GHz, IEEE 802.15.4 and ZigBee application [5]. It is standard enhancement type 8051 that can establish powerful network nodes at 
very low total cost of materials. Combined with Texas instruments industry leading gold unit ZigBee protocol Stack (Z - Stack TM), it provides a powerful and complete ZigBee solution. CC2530 has different operating modes, making it especially suitable for systems with low power consumption requirements. The short transition time between the running patterns further ensures low energy consumption. The CC2530 circuit is shown in figure 3.

The TGS2600 sensor is made up of a metal oxide sensitive chip with metal aluminum substrate and a complete heater. When In detecting the gas, the sensor's conductivity depends on the concentration of gases in the air. TGS2600 has a high sensitivity to low concentrations of cigarette pollutants in the air, such as $\mathrm{H}_{2}$, CO, or cooking. Sensors can detect several ppm levels of $\mathrm{H}_{2}$. This sensor uses relative value detection to achieve more control of human perception. That makes clean air as a baseline to measure the extent of air pollution through the relative sensor resistance to the clean air.

The temperature and humidity sensor module is composed of temperature and humidity sensor and signal conditioning circuit. The system adopts the temperature and humidity compound sensor DHT11 which has calibrated digital signal output. The sensor mainly consists of a resistive sensor and an NTC temperature sensor, which is connected to a high-performance 8 single chip. So it has the advantages of super quick response, super small size, very low power consumption, strong anti-jamming ability, high price ratio, and the long signal transmission distance that can reach up to 20 meters, and greatly improves the reliability and ease of the system.

\subsection{Master monitoring system hardware}

As the control center of the system, system control host needs the power supply module, ZigBee wireless sensor network communication module, CPU module, information display module, information input module, control output module, et

LCD information display module: LCD12864 LCD display module is a Chinese character graphic display module with $128 * 64$ dot matrix. It can display Chinese characters and graphics with 8192 Chinese characters (16*16 dot matrix), 128 characters (8*16 dot matrix) and 64*256 dot matrix to display RAM (GDRAM). The current indoor temperature, humidity and air quality are mainly displayed.

Alarm display output module: Alarm circuit consists of PNP triode and speaker. Alarm should be issued when ambient temperature is not set in temperature range.

\section{System software}

\subsection{Detection slave system software}

Detection slave mainly collect information from the sensor module, and sent to the master. The flow chart of slave is shown in figure 5.

\subsection{Detection master system software}

The flow chart of master is shown in figure 6. It mainly accomplishes the following tasks.

(1) Initialization. Initialize the working parameters in memory to show the system initialization state.

(2) Read temperature and smoke sensor parameters.

(3) Determine whether the set temperature been exceeded. If the temperature limit is exceeded, the cooling module will be activated. While if not, then continue to judge.

(4) Determine the concentration of air pollution. If the pollution concentration exceeds the limit, then automatically grades and the alarm module will be started.

(5) Determine if it is below the set temperature. Start the heating module if the temperature is below the lower one. 
(6) The load module is activated when the temperature is set and the air concentration is set.

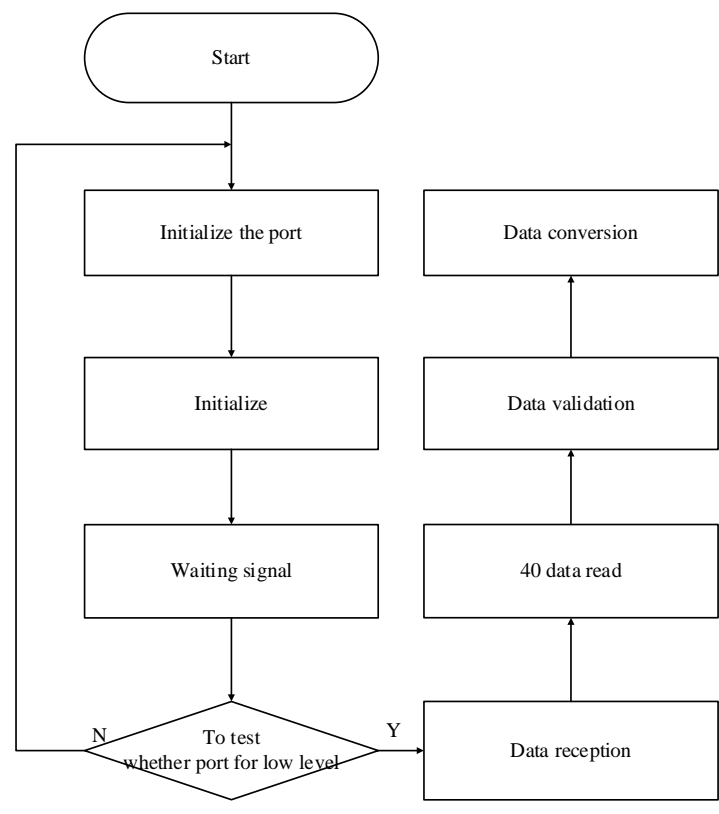

Fig.5 Flow chart of slave

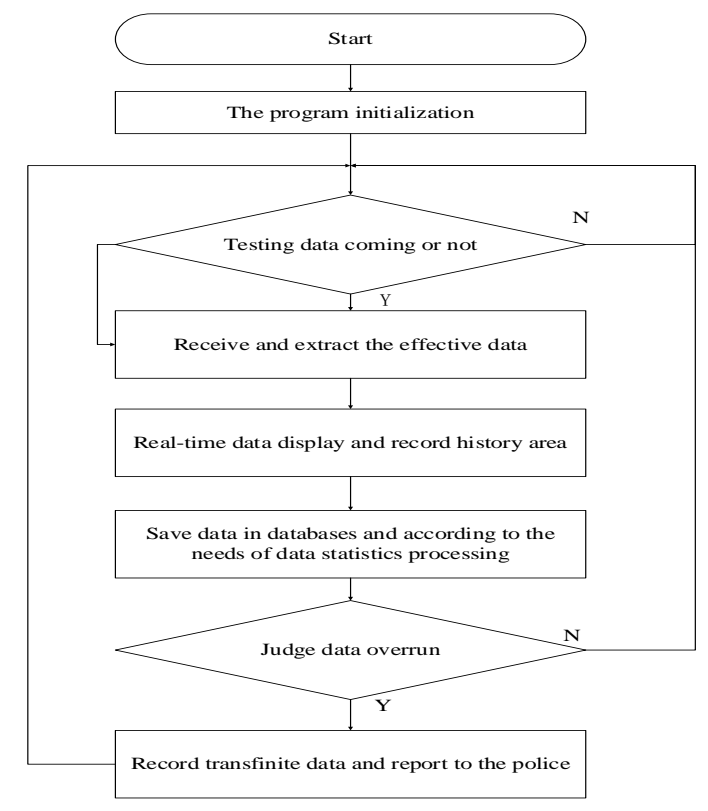

Fig.6 Flow chart of master

\section{Experimental results}

\subsection{System hardware}

It includes master and slave as the show in figure 7:

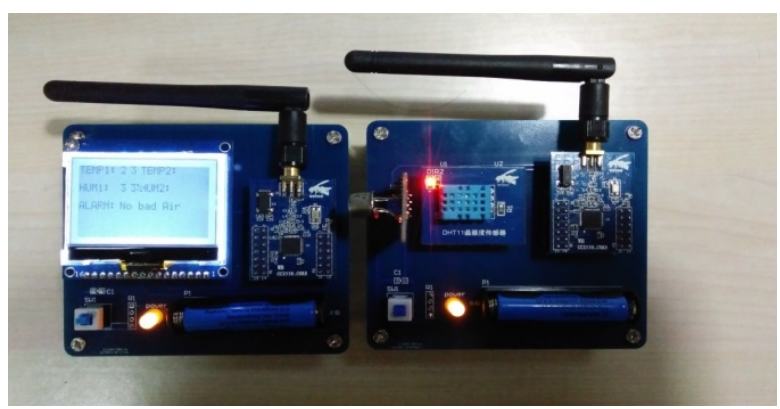

Fig.7 System debugging

\subsection{Results in data and air pollution}

TGS2600 sensor is able to detect the smell of pollutants in the air and monitor changes in air quality. The sensitivity of the TGS2600 sensor indicates that its sensitivity is affected by air temperature humidity and the concentration of pollutants in the air. The system of temperature and humidity detection module and a temperature and humidity control module, temperature and humidity can be controlled within a certain range, so we can ignore the influence of the air temperature and humidity on the sensitivity. The level of indoor air pollution can be determined by the sensor's sensitivity range. The sensitivity of the sensor is that the resistance is more than Rs/Ro, and the Rs and Ro are defined as follows: Rs indicates that the sensor's resistance values when air pollution is in the room at a certain temperature and humidity; Ro indicates the sensor resistance of the indoor air at the same temperature and humidity. At 20 degrees Celsius and 65\% humidity, we did the experiment. We first blasted low pollution gas and then removed it, finding that system test results are as the same as expected, instructing that system has entered a stable state. Next, we entried into the formally experiment. Blasting a lot of pollution gas, we collected gas information and system program conversion through TGS200 module, and finally got the corresponding gas concentration information. The results of the experiment are shown in table 1. 
Table 1 Levels of pollutant and air pollution

\begin{tabular}{|l|l|l|l|}
\hline Pollutants[ppm] & Rs/Ro & Experiments & Display \\
\hline $1.5 \sim 2.5$ & $0.60 \sim 0.50$ & $\begin{array}{l}\text { No pollution } \\
\text { gases }\end{array}$ & No pollution \\
\hline $2.5 \sim 4.0$ & $0.45 \sim 0.50$ & $\begin{array}{l}\text { Blast low } \\
\text { pollution gas }\end{array}$ & Slight pollution \\
\hline $4.0 \sim 5.0$ & $0.40 \sim 0.60$ & $\begin{array}{l}\text { Remove } \\
\text { pollution gas }\end{array}$ & $\begin{array}{l}\text { Slight pollution } \rightarrow \text { no } \\
\text { pollution }\end{array}$ \\
\hline $2.5 \sim 8.0$ & $0.35 \sim 0.50$ & $\begin{array}{l}\text { Blast a lot of } \\
\text { pollution gas }\end{array}$ & $\begin{array}{l}\text { Slight pollution } \rightarrow \text { heavy } \\
\text { pollution }\end{array}$ \\
\hline $1.5 \sim 8.0$ & $0.35 \sim 0.60$ & $\begin{array}{l}\text { Remove } \\
\text { pollution gas }\end{array}$ & $\begin{array}{l}\text { Heavy pollution } \rightarrow \text { medium } \\
\text { pollution } \rightarrow \text { slight pollution } \rightarrow \\
\text { no pollution }\end{array}$ \\
\hline
\end{tabular}

\section{Conclusion}

This project designs a set of multipoint real-time monitoring system of household air quality based on ZigBee. It monitors household air quality in real time and alerts the alert based on the detected data. The experimental results show that the collected data is accurate and effective, and achieves the desired design effect and purpose. Nowadays people pay attention to the household air quality, so the study of this article has certain reference effect to the study of the future household air quality [6].

\section{Acknowledgements}

This work is supported by National Undergraduate Innovational Experimentation Program (201410060044).

\section{References}

[1] Wang Wen-bo, Zhao Lu-pei, Mei Xiao-dong, Wang Biao, Lu Ge-yu. Design of wireless sensor networks air monitoring system based on ZigBee[J].Transducer and Microsystem Technologies,2014.33 (2):83-86.

[2] Gen Jun-tao, Zhou Xiao-jia, Zhang Bing-jie. An Atmospheric Environment Monitor System Based on Wireless Sensor Network[J].Journal of Xihua University,2007,26 (4):44-46.

[3] Chang Chao, Xian Xiao-dong, Hu Ying. Design of Precision Agricultural Remote Environment Monitoring System Based on WSN[J].Chinese Journal of sensors and Actuators,2011,24 (6) :879-883.

[4] Wang Zhong-sheng, Cao Mei. Design and implementation of serial monitoring software based on ZigBee[J]. Electronic Design Engineering,2011,19 (14):13-15.

[5] Tang Hui-qiang, Li Chao. Design of the lithium battery detection system based on CC2530[J]. Control and Instruments in Chemical Industry,2012,38(3):353-355.

[6] Wen Hou-ming, Lin Xiao-kang. Based on the ZigBee Protocol Intelligent Household Detection System Design[J].Audio Engineering,2013,37(11) :74-80. 